Neurosurg Focus 11 (5):Introduction, 2001, Click here to return to Table of Contents

\title{
Overview of Management Schemes for Intracranial Arteriovenous Malformations.
}

\author{
Neurosurgical Focus, November 2001 \\ Volume 11, Issue 5 \\ Topic Editor: Philip E. Stieg, M.D., Ph.D. \\ Weill Medical College of Cornell University, and New York Presbyterian Hospital, New York, New York
}

The first documented complete excision of a cerebral arteriovenous malformation (AVM) was performed in 1889 by the French surgeon Jewels Amil Pean. Amazingly, this was performed prior to the introduction of cerebral angiography by Egas Moniz in 1928. Subsequently, Professor Olivecrona was considered the pioneer who developed surgery as an acceptable treatment for intracranial AVMs. He is responsible for introducing many of the surgical approaches used for the resection of AVMs. Most notably, however, he recognized that resection of the venous drainage system had to take place at the end of the procedure. After his retirement, technological advancements, including the microscope and bipolar cautery, led to significant improvement in microsurgical techniques. Professor Yaşargil played a critical role in the introduction of the operating microscope for cerebrovascular surgery. In addition, he was one of the first individuals to propose the use of presurgical embolization. Over the past decade, a mutlimodality management scheme for cerebral AVMs has been developed. Combinations of microsurgery, radiosurgery, and endovascular approaches have been used to provide more effective, less morbid, and complete excision.

In addition to the improvements in microsurgical technique, many diagnostic and anesthetic advances have also facilitated our ability to resect difficult lesions. The advent of magnetic resonance (MR) imaging, functional MR imaging, computerized tomography angiography, superselective angiography with Wada testing, and neuropsychological evaluation have all improved our ability to delineate the margins of an AVM and improve treatmentrelated outcomes by using multimodal schemes.

This issue of Neurosurgical Focus will provide an overview of specific topics important to the management of intracranial AVMs. The epidemiology and natural history of these lesions will be reviewed initially. In the second paper the authors discuss the diagnostic approaches and the evaluation of functionally eloquent cortex.
In the third article the authors apply these diagnostic tools to the next level of evaluation in which the surgeon has to grade the AVM and determine the surgery-related risk. In addition, these authors review the process in which surgeons plan their surgical approach after having carefully evaluated the lesions. In the fourth article the authors review current concepts in neuropsychiatric evaluation. This modality may be used preoperatively to gain a better understanding of the functional cortex that surrounds the AVM. As expected, this information is predictive of surgery-related results and has an effect on our treatment recommendations. The authors of the fifth article review the current anesthetic approaches used pre-, intra-, and postoperatively. In the sixth article the writers discuss the use of embolization as an adjuvant to microsurgical resection. In particular, the use of silk sutures is compared with other embolic agents. Finally, the authors of the last article describe a decision analysis scheme, which can be used for selecting the appropriate treatment for small intracranial AVMs.

It is clear from the literature that AVMs that are difficult to treat with surgery are usually also challenging in endovascular and radiosurgery. Thus, each case has to be carefully assessed by a team of physicians to determine a treatment plan that combines the advantages of each modality and keeps the overall complication rate at a minimum. Because the treatment plan is highly dependent on the individual patient characteristics, it is not possible to formulate a general treatment scheme involving all the potential therapeutic options. It is unlikely that there will be a controlled study in which multimodality treatment of AVMs are compared with conventional monotherapy. It is the general consensus of most cerebrovascular neurosurgeons, however, that a multidisciplinary team ensures the most effective approach to the treatment of these complex lesions. 